\title{
Swapping of interaction partners with ATG5 for autophagosome maturation
}

\author{
Jun Hoe Kim E Hyun Kyu Song ${ }^{*}$ \\ Division of Life Sciences, Korea University, Seoul 136-713, Korea
}

\begin{abstract}
Autophagy is a tightly regulated lysosome-mediated catabolic process in eukaryotes that maintains cellular homeostasis. A distinguishable feature of autophagy is the formation of double-membrane structures, autophagosome, which envelopes the intracellular cargoes and finally degrades them by fusion with lysosomes. So far, many structures of Atg proteins working on the autophagosome formation have been reported, however those involved in autophagosome maturation, a fusion with lysosome, are relatively unknown. One of the molecules in autophagosome maturation, TECPR1, has been identified and recently, structural studies on both ATG5-TECPR1 and ATG5-ATG16L1 complexes revealed that TECPR1 and ATG16L1 share the same binding site on ATG5. These results, in combination with supporting biochemical and cellular biological data, provide an insight into a model for swapping ATG5 partners for autophagosome maturation. [BMB Reports 2015; 48(3): 129-130]
\end{abstract}

The formation of autophagosome requires ubiquitin-like (Ubl) conjugation steps. Atg7 is a unique E1-like enzyme that activates the Ubl molecules, Atg8 and Atg12, in autophagy. Atg8 and Atg12 are conjugated with different E2-like enzymes, Atg3 and Atg10, respectively. In the autophagy system, there is only an E3-like enzyme, Atg12 Atg5-Atg16 complex for lipid modification of Atg8 (LC3s and GABARAPs in mammals) in contrast to numerous E3 ligases in the ubiquitin-proteasome path-

*Corresponding author. E-mail: hksong@korea.ac.kr

http://dx.doi.org/10.5483/BMBRep.2015.48.3.048

Received 19 March 2015

Keywords: ATG5, ATG16L1, Crystal structure, Lysosome fusion, TECPR1

Abbreviations: AFIM, Atg5(five)-interacting motif; AIR, ATG12 ATG5-interacting region; ATG, autophagy-related; ATG16N69, ATG16L1 N-terminal 69 residues; TECAIR, TECPR1 AIR; TECPR1, tectonin $\beta$-propeller repeat containing 1 ; Ubl, ubiquitin-like

Perspective to: Jun Hoe Kim et al (2015), Insights into autophagosome maturation revealed by the structures of ATG5 with its interacting partners, Autophagy, 11(1):75-87, doi: 10.4161/15548627. 2014.984276 way $($ ' $\sim$ ' depicts the covalent bonds, while ' - ' depicts noncovalent bonds). Therefore, the primary enzymatic role of Atg5 is accelerating the phophatidylethanolamine attachment to the C-terminus of Atg8, which is a critical step for autophagosome formation. However, human ATG5 plays additional roles in other autophagic processes. The ATG12 ATG5-ATG16L1 complex locates at autophagosomal membrane before the autophagome fuses with lysosomes (Fig. 1). A couple of years ago, TECPR 1 (tectonin $\beta$-propeller repeat containing 1 ), a lysosomal membrane bound protein, has been characterized as an ATG5 interacting molecule. Recently, we determined the structures of human ATG5 in complex with the binding region of its binding partners, ATG16L1 (ATG16N69) and TECPR1 (TECAIR). A portion of the $\mathrm{N}$-terminal coiled coil region of ATG16L1 forms a complex with ATG5 and a central region called AIR (ATG12-ATG5 interacting region) also does. According to our structural analysis, an amino acid sequence, $\mathrm{W}-\mathrm{x}_{3}-\mathrm{I}-\mathrm{x}_{3}-\mathrm{L}-\mathrm{x}_{2}-\mathrm{R}-\mathrm{x}_{3}-\mathrm{Q} / \mathrm{E}$ (' $\mathrm{x}^{\prime}$ 'stands for interval with any kinds of amino acids) of ATG16L1 or TECPR1 was found to be a key factor for ATG5 binding and exists as an $\alpha$-helical structure. Its conservation from yeast to metazoan was confirmed by sequence alignment and thus we coined it as AFIM (ATG5[five]-interacting motif). The significance of AFIM was confirmed by mammalian two-hybrid assays with the structure-based mutants of ATG16L1 and TECPR1. Furthermore, accumulation of autophagosomes in cells caused by the defect of lysosomal fusion step was observed by autophagic puncta monitored with GFP-labeled LC3 and also electron microscopic images.

Our two crystal structures clearly show that ATG5 cannot accommodate both ATG16L1 and TECPR1 simultaneously. According to the previous studies by Ogawa et al., ATG5, ATG16L1, and TECPR1 can all colocalize in the phagophore. However, our structural and mutagenetic studies support the experimental result of Zhong and colleagues, who showed that TECPR1 cannot exist with ATG16L1. The structural superposition of AFIM in both ATG16L1 and TECPR1 shows them to be nearly identical, but the structure outside the AFIM is markedly different (Fig. 1). ATG16L1 possesses a continuous $\alpha$-helical structure toward the C-terminal direction outside the AFIM while TECPR1 has a serpentine loop structure with extensive interaction with ATG5. The interface area in the complex between ATG5 and TECAIR is almost $200 \AA^{2}$ more than 


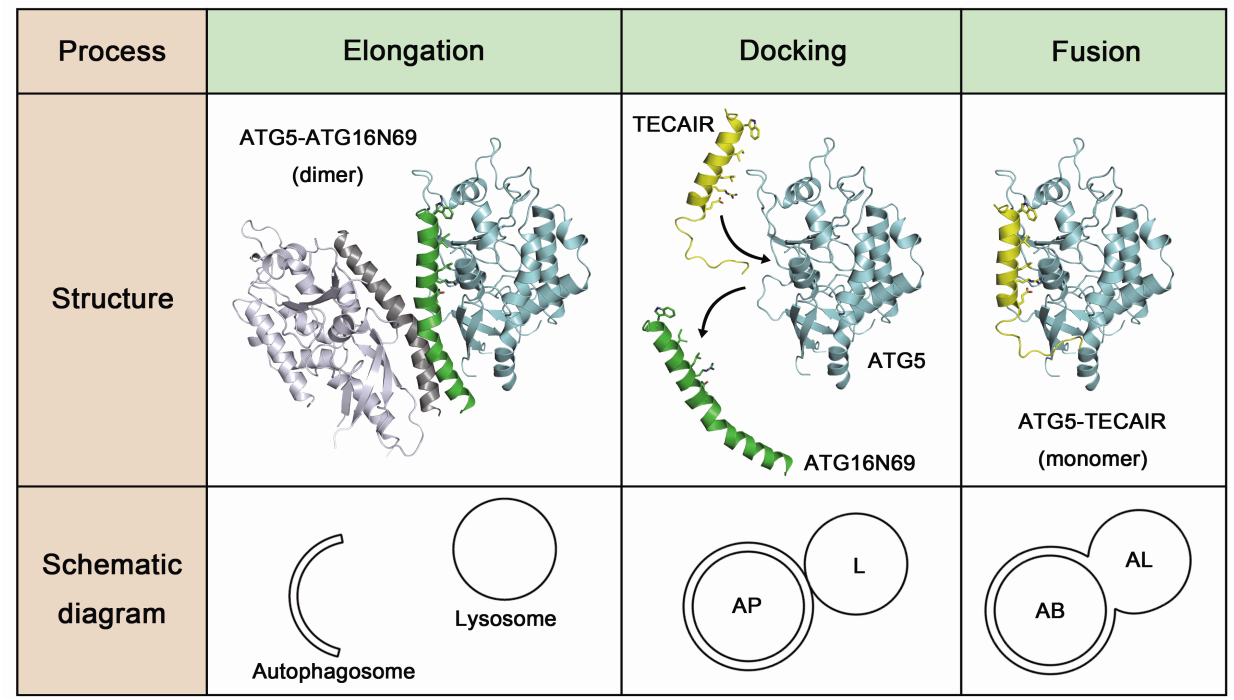

Fig. 1. A model for autophagosome maturation by swapping ATG5 interacting partners. During autophagosome elongation, ATG16N69 (green) binds to ATG5 (cyan) with its conserved AFIM (protruding residues as a stick model) and forms ATG5-ATG16N69 dimer. After autophagosome formation is completed, the autophagosome docks lysosomes in order to degrade cellular cargoes by fusion. In this stage, the surrounding acidic condition drives ATG5 to change its binding partner from ATG16N69 to TECAIR (yellow). Once TECAIR binds to ATG5, its unstructured tail region gives tighter binding forces to make the ATG5-TECAIR complex. This monomeric protein complex increases the chances of interaction among other membrane fusion factors and finally autolysosome is generated (AP, autophagosome; L, lysosome; $\mathrm{AB}$, autophagic body; $\mathrm{AL}$, autolysosome).

that between ATG5 and ATG16N69. Clearly, TECPR1 shows more extensive interaction with ATG5 via its C-terminal unstructured region, suggesting that during autophagosome maturation, ATG5 swaps its interacting partner from ATG16L1 to TECPR1 (Fig. 1).

The in vitro binding constants between ATG5 and its binding partners were measured using a surface plasmon resonance technique. Unexpectedly, the binding affinity is greatly affected by different $\mathrm{pH}$ values and intriguingly, both ATG16L1 and TECPR1 peptides showed the enhanced binding affinity to ATG5 at lower $\mathrm{pH}$. There might be a possibility that the cytosolic $\mathrm{pH}$, especially near the lysosome, is relatively acidic and the ATG5 and TECPR1 interaction must be stronger at this acidic condition surrounding those proteins. Our data showed that the binding affinity between ATG5 and ATG16L1 is tighter than that between ATG5 and TECPR1 at neural $\mathrm{pH}$, however it becomes quite similar at acidic pHs. Particularly, the dissociation rate constant $\left(k_{d}\right)$ between ATG 5 and TECAIR peptide is lower than that between ATG5 and ATG16N69 peptide, suggesting that swapping the interacting partner from ATG16L1 to
TECPR1 must be a unidirectional process.

The structurally determined ATG16N69 is a dimer (Fig. 1) and the exact oligomeric nature of full-length ATG16L1 in cells is different. The TECPR1 possesses a lipid binding PH domain followed by TECAIR and several tectonin $\beta$-propeller repeats, whose exact role is not yet identified. The structural study and the binding affinity measurements have been done with a small domain and/or minimal length of peptides in both ATG5 interacting partners due to technical difficulty, thus the behavior of full-length proteins need to be further investigated. However, our current structural, biochemical, and cell biology data for ATG16L1 and TECPR1 provide a good and detailed molecular dissection of the autophagosome maturation, which will help to guide future investigations.

\section{ACKNOWLEDGEMENTS}

This work was supported by grants from National Research Foundation of Korea (NRF-2011-0028168 to HKS; NRF-2012Global PhD Fellowship to JHK). 